\title{
Studies on Rich Media and Related Knowledge Organization
}

\author{
Zhang Yunliang \\ Institute of Scientific \& Technical Information of China; \\ Key Laboratory of Rich-media Knowledge Organization \\ and Service of Digital Publishing Content \\ Beijing 100038, China \\ zhangyl@istic.ac.cn
}

\author{
Wang Li \\ Institute of Scientific \& Technical Information of China; \\ Key Laboratory of Rich-media Knowledge Organization \\ and Service of Digital Publishing Content \\ Beijing 100038, China \\ wl@istic.ac.cn
}

\begin{abstract}
Rich media is introduced and analyzed from the concept itself, typical applications in different fields, the characteristics and influence in two sizes. Knowledge organization and traditional knowledge organization systems are also analyzed and most of them are suitable for rich media. In this paper, 9 typical applications are reorganized from four dimensions of contents, time, space and human. This paper also points out the feature representation and mapping problems in rich media knowledge organization work.
\end{abstract}

Keywords—rich media, knowledge organization, application, dimension

\section{INTRODUCTION}

Within a long period, people get their information and knowledge mainly through books, journals and newspapers, but sometimes there are some ambiguity on these paper publications and it's not enough to understand the exact meaning of the authors. Then came the broadcasting stations and television stations, people could get richer information, there are no interaction between the information creators and the audience yet. Now there are some advances to improve the current situation. The internet, mobile devices, and the high speed communication techniques such as 4G, 5G and satellite communication change the world a lot. With these facilities, everyone can produce his own images, audio, and video and share with friends or other people all around the world conveniently. New media can promote more equal, more interactive, more intuitional and richer information spreading patterns. Some researchers named it rich media.

From Google Books Ngram Viewer, there are some related Ngrams as early as 1904, but after check papers from Web of Knowledge, It was found that at earlier time, it's only an Ngram, but not a phrase. In 1990s, there are some papers discussed the richness of the media. But maybe the earliest discussion of this concept emerge at 1993 or so and studied by Brown [1]. When 1999, the EyeWonder [2] was founded and rich media was proposed and developed in advertisement field. Usually, rich media is equals to interactive media, which normally refers to products and services on digital computer-based systems which respond to the user's actions by presenting content such as text, moving image, animation, video, audio, and video games[3]. It is defined from a technical view, and first interactive technique with computer was developed in 1958. From the literatures, it is found that most papers about the rich media are published after 1999. And when turn back to the publishing field, rich media affect a lot of it, especially digital publishing. People still have a lot of paper publications, but you can see some QR Codes or Two-dimensional Codes dotted within texts, if you scan a code, it will link to an image, a piece of audio or video, and you can get richer information than only from the texts. Sometimes it can even link to some services such as course tests or peer-to-peer guidance. In education scenes, there are talking pen, if a child put the pen onto some specific part of a specific book, the pen will read a story or sing a song. AR technology also has been used in the education books. For a long time electrical book is invented, which can be read through electric reader such as kindle or apps of the smartphones.

In fact, with more and more media types included in the rich media, there are great demands to organize the different media. So that the content providers can use these media to reform different knowledge services for different end users.

\section{DEFINE RICH MEDIA}

From Techpedia.com [4], there is a definition: The term rich media refers to a specific type of installations on the Web that are more functional and interactive. The core definition of rich media is that it is a Web page ad that contains video or interactive features. It is a very narrow definition and limited to some computer techniques. In fact rich media involves publishing, advertisement, entertainment and livelihood.

In this paper, the concept are discussed with reductionism and some examples are used to explain its application in different scenes.

\section{A. Media and Rich and Related Concepts}

From the view of reductionism, the concept is combined with media and rich, so both atom concepts should be considered.

Media in fact is a concept in a large scope, sometimes are very widely used in chemistry and other disciplines. In this paper, this concept could be studied from four aspects, firstly media means the communication channels of the information, secondly it means the organizations that produce these contents, thirdly it also means the storages of the information like paper, disk, magnetic tape and even internet, lastly it means different forms such as texts, images, audio, video and different formats of specific form. Usually techniques focus on storage and formats, but organizations and channels are 
very important.

Rich means that there are a lot of formats of media process by different organizations in different storages and spread from different channels, which is shown in Fig. 1. And sometimes rich means redundant. But it is a relative concept, for example, when the first silent film emerged, most people thought it was very rich but when sound film came, silent film wasn't rich any more.

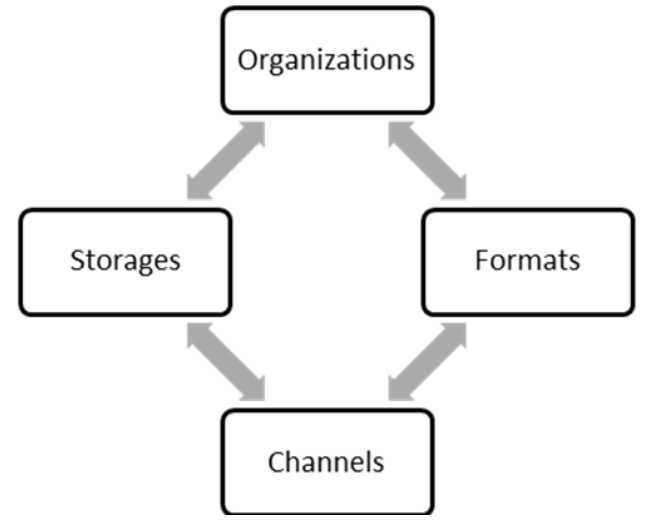

Fig. 1. The Different Aspects of Media and Combine to Rich Media

\section{B. Publishing Applications}

Royal Society of Chemistry (RSC) firstly realize the Enhanced HTML article and then renamed as rich HTML article, in which hypertext links are used to expand the related knowledge of science articles. Then publishers such as Elsevier, Wiley and PLoS try to implement Article of the Future, Smart Article, and Anywhere Article etc. [5]

In scientific papers, usually there are some programmes to calculate and explain the results of experiments, the normal articles even the enhanced articles can't express the enormous data and results easily. So there are some solutions. Bret Victor proposed a scientific paper that had been redesigned as a sequence of illustrations with captions [6], and everyone can read the reformed paper and adjust the parameters and see the results immediately. There are also software to support these work, one commercial software is Mathematica's notebook, and another software is Jupyter notebook[7], which once called IPython, and nowadays, it can support a lot of scientific programming language such as C, R , Ruby, Javascript, or Julia etc. besides Python.

Institute of Geographical Sciences and Natural Resources Research, Chinese Academy of Sciences developed a new scientific data publishing service, which is called Global Change Research Data Publishing \& Repository (GCRDP\&R) [8], which are mainly about geography, now includes 471 datasets that takes up more than 221.75 Gigabytes disk space, and contributed by 779 authors form 12 countries. The Integrated Digitized Biocollections (iDigBio) [9] is funded by the National Science Foundation of USA and developed a team from the University of Florida and Florida State University and so on, and its goal is to integrate the biology related collections such as filed records, photos, specimens, geological information and 3-D images and so on from 92 institutions from 45 states of USA. Now it has more than 115 million Specimen Records, 26 million Media Records. Science Data Bank (SDB) [10] is also an online scientific data publishing platform, now it has 149 datasets, and more widely data fields are covered. As
GCRDP\&R and iDigBio are vertical scientific data digital publishing application, SDB is a comprehensive scientific data digital publishing application. Of course the library also can provide services with these publications or reformed ones [11].

\section{Advertisement Applications}

The mainstream advertisement on the internet are mainly rich media nowadays. There are different types of internet advertisement, once the static images are widely used, but now more and more dynamic media are used, especially when the shockwave flash and other techniques to show and link rich media emerge. The advertisement might on the banner, the button, the floating icon, or the full screen animated advertisement, especially in some special period to promote some products or celebrate holidays.

The elevator advertisement or the shopping terminal advertisement are richer and more automotive, when you enter the special area and the equipment detect you, it will show the advertisements, usually the advertisements are composed with both video and posters[12].

\section{Entertainment Applications}

As far one of the most popular entertainment activities is short video, there are lots of Apps such as TikTok and MeiPai. These apps are attractive because they combine the music, the video of end users and some animations together, and they are really rich media. Combine with these P2P short video and most online B2C video providers, such as Tencent video, iqiyi, Youku and so on, sometimes there are advertisement board in the video and sometimes there are two-dimensional codes or shopping links embedded within the video, they are also rich.

\section{E. Livelihood Applications}

Rich media are also widely used in our lives. One typical application is Navigation. Navigation combine with texts, maps, video and voices together. There are a lot of redundancy in Navigation applications. For most occasions, only the voice guide or the video guide is enough to let you know where to go and how to go. But in some cases, you need to use both of these information and look at the real road condition and to know how to go.

When you visit some review website, group buy website or housing agencies, you can browse the texts and the images, sometimes you can also see the videos and even some AR videos, so you can get a more comprehensive information about the shops or the houses. There are also some AR fitting room apps, that you can fit different clothes very quickly without going to the shopping malls.

So rich media become popular in clothing, food, housing and transportation and all other aspects of life.

\section{CHARACTERISTICS AND INFLUENCE OF RICH MEDIA}

\section{A. The Typical Characteristics}

There are three typical characteristics of rich media. The first is interactivity, from Techopedia [4] rich media is more functional and interactive, the rich idea is almost equals to interactive media, so interactivity is very important to the rich media. There are lots of contents in the rich media, so the operation of the user can change to different scenarios. 
For example, when use navigation apps, you can choose different route to fit your priority of time, distance or other requests. The second is real-time, if the time changed, the media information is not useful, of course navigation is an example, and the shop advertisement is the same, since if you are in the shop, the advertisement will encourage you to buy something you don't want to buy before. The third is content augmentation, since rich media sometimes is redundant, so the content is augmented with different types of media. The rich media also has an augmented character from the technology advance, for example, once when you watch a $160 \mathrm{P}$ or $320 \mathrm{P}$ video, usually you see many color blocks and can't identify the scenarios clearly, but when it evolves to 480P, 720P, 1080P, $2 \mathrm{~K}, 4 \mathrm{~K}$ and even $8 \mathrm{~K}$, you can see much more things from the video. These are typical characteristics of rich media but it doesn't mean rich media have to have all three characteristics.

\section{B. Influence: Two-side}

With rich media, people can get information almost without loss. Why does the information lose? The channel and distances. Usually we get the information not from the original author, with a paraphrase, there will be little difference, but after a lot of paraphrases, it will lost a lot of information, it can be roughly illustrated by Equation (1). But if rich media are used, since it have different media types, every time when information is transferred, different type media can verify and get right information from redundant media, and usually there almost no information loss, as is roughly shown in Equation (2). In the two formulas, we suppose that every media type will lose $10 \%$ after one transformation, and there are three media types in rich media.

$$
0.9 \times 0.9 \times 0.9 \times \cdots \cdots \rightarrow 0
$$

$$
\begin{array}{r|r|r}
0.9 & 0.9 \\
0.9 & 0.9 \\
0.9 & 0.9 & 0.9 \\
0.9 & 0.9
\end{array} \mid \times \cdots \cdots \rightarrow 1
$$

But as is known that television video can affect the imagination and creativity than audio and texts, the rich media can provide more information than video, so maybe it will affect the imagination and creativity more than the video. And it will affect the evolution of information and knowledge and decrease its diversity, and it is not good for the whole people's development.

\section{KNOWLEDGE ORGANIZATION IN RICH MEDIA}

\section{A. Applicability}

Knowledge organization are firstly used in literatures. But when you review its definition and methods, you will find that it isn't limited to [13]. Whatever the media types are, the information need to be arranged, processed, represented and controlled as knowledge objects. So knowledge organization is a widely applicable concept, even when the knowledge is in the form of rich media. To do knowledge organization work, you need knowledge organization systems. Let's review the different types of knowledge organization systems. Usually there are term lists, hierarchical organization and network organization. Term lists are the most universal and widely used, of course you can describe any rich media with a few terms. And the hierarchical organization such as classifications and taxonomies also have great flexibility, if the hierarchical organization covered the dimensions of the rich media, the media can be easily classified, otherwise it is very difficult. For example, it will confuse you if you want to classify herbal medicine into a medicine system for diseases of different organs. For network organization it's a little different, knowledge organization systems like ontologies and vocabulary systems are similar to hierarchical organization, while the thesauri should change a lot to fit the rich media, which means except for the master thesaurus about the contents, the auxiliary lists for entities like countries, nations, races and languages should be emphasized.

\section{B. The Dimensions of Organization}

Rich media can be organized from different dimensions. Every application can organize rich media from one or more dimensions of contents, time, space and human and so on. Some typical application are analyzed in Figure 2. Usually every application, especially some fusion knowledge service with different media types, is organized by contents. The subtitles of videos and time flow charts are organized with time. Electronic tour guide organize texts, voices, video together and can be activated by location, though some are activated by two-dimensional codes. Social network services are activated by human, usually friends or human with same interests are gathered and they can share with rich media. Navigation is an application served from both time and space dimensions, the navigation rich media must be provided at right place and in right time. Barrage mainly works at right time with the crowd having the same interests. Location based advertisements should consider the space and human, to provide reachable promotion information. Smart Advertisements can recommends from time, space and human. Game, especially online games, usually provides specific people at the same time with location relationship like the same city.

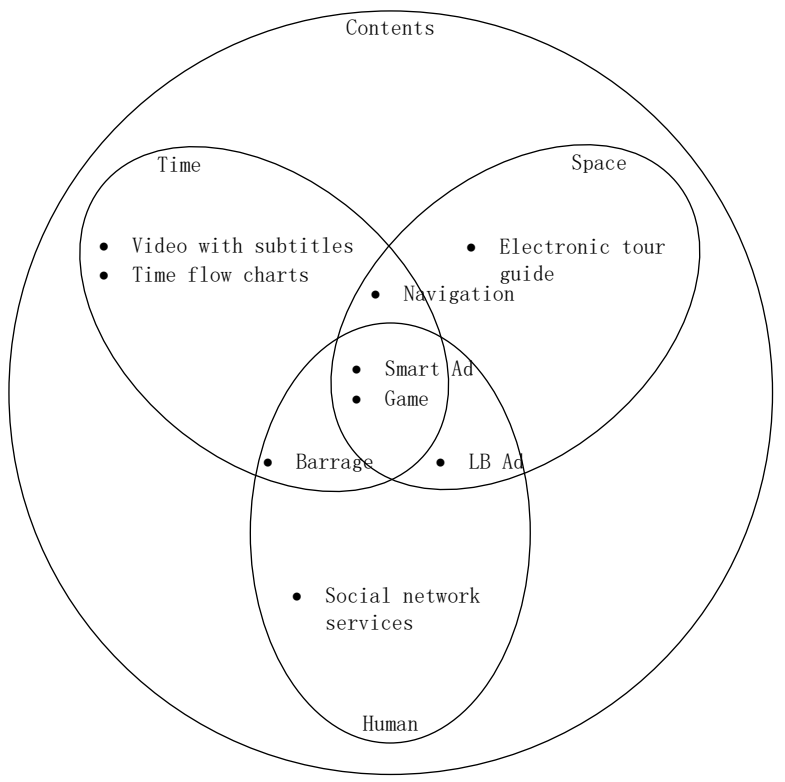

Fig. 2. The Different Aspects of Media and Combine to Rich Media 


\section{Key Problems}

Rich media can be organized from different dimensions. Every application can organize rich media from one or more dimensions of contents, time, space and human and so on. Some typical application are analyzed in Figure 2. Usually every application, especially some fusion knowledge service with different media types, is organized by contents. The subtitles of videos and time flow charts are organized with time. Electronic tour guide organize texts, voices, video together and can be activated by location, though some are activated by two-dimensional codes. Social network services are activated by human, usually friends or human with same interests are gathered and they can share with rich media. Navigation is an application served from both time and space dimensions, the navigation rich media must be provided at right place and in right time. Barrage mainly works at right time with the crowd having the same interests. Location based advertisements should consider the space and human, to provide reachable promotion information. Smart Advertisements can recommends from time, space and human. Game, especially online games, usually provides specific people at the same time with location relationship like the same city.

\section{KNOWLEDGE ORGANIZATION IN Rich MEDiA}

Rich media can prove more sufficient and detailed information and knowledge, so it could be used widely in different fields. Since rich media has distinct characteristics and knowledge services could be various, rich media can be analyzed and organized from different dimensions such as contents, time, space and human. Most knowledge organization system can be easily used for rich media, but some should be revised and key problems such as feature representation and mapping should be resolved in the future work.

\section{ACKNOWLEDGMENT}

This research was financially supported by ISTIC Key
Project Program (Grant No. ZD2018-07), CKCEST Project Program (Grant No. CKCEST-2018-1-26) and National Digital Composite Publishing System Project (Grant No. XWCB-ZDGC-FHCB/28).The authors also gratefully acknowledge the helpful comments and suggestions of the reviewers, which have improved the presentation.

\section{REFERENCES}

[1] Brown, P .The Ethics and Aesthetics of Rich Media Computer Human Interfaces[C]. Maurer, H. Proceeding of World Conference on Educational Multimedia and Hypermedia (Ed-Media 93)Educational Multimedia and Hypermedia Annual, 1993,PP603

[2] EyeWonder[EB/OL].https://www.crunchbase.com/organization/eyew onder\#section-locked-charts, [2018-11-10].

[3] Interactive media [EB/OL]. https://en.wikipedia.org/wiki/Interactive_media, (2018-09-07) [201811-10].

[4] Rich Media [EB/OL]. https://www.techopedia.com/definition/9202/rich-media, [2018-11$10]$.

[5] SONG Ningyuan, WANG Xiaoguang. Comparisonand analysis of enhanced publication models[J](In Chinese). Chinese Journal of Scientific and Technical Periodicals,2017,(7):587-592

[6] Scientific Communication As Sequential Art [EB/OL]. http://worrydream.com/ScientificCommunicationAsSequentialArt/, (2011-05-24) [2018-11-10].

[7] James Somers.The Scientific Paper Is Obsolete[EB/OL]. https://www.theatlantic.com/science/archive/2018/04/the-scientificpaper-is-obsolete/556676/, (2018-04-05) [2018-11-10].

[8] Global Change Research Data Publishing \& Repository [EB/OL]. ttp://www.geodoi.ac.cn/, [2018-11-10].

[9] iDigBio[EB/OL]. https://www.idigbio.org/, [2018-11-10].

[10] Science Data Bank[EB/OL].http://www.sciencedb.cn/, [2018-11-10].

[11] QIN shuying, WU xuezhi, GUAN jingxia. Elaborate Tries on Academic Library Reading Promotion at Omnimedia Times (in Chinese)[J]. TECHNOLOGY INTELLIGENCE ENGINEERING, 2015, 1(1):103-111.

[12] Different Advertisement (in Chinese) [EB/OL]. https://www.idigbio.org/, [2018-11-10].

[13] ZENG wen, LI ying, HAN hongqi, et al. Study on the Method of Data Organization and Management (in Chinese) [J]. TECHNOLOGY INTELLIGENCE ENGINEERING, 2016, 2(1):109-113. 